\title{
Improved Bounds for a Deterministic Sublinear-Time Sparse Fourier Algorithm
}

\author{
M. A. Iwen \\ Department of Mathematics \\ University of Michigan - Ann Arbor \\ Ann Arbor, MI 48109-1043 \\ Email: markiwen@umich.edu
}

\author{
C. V. Spencer \\ Department of Mathematics \\ University of Michigan - Ann Arbor \\ Ann Arbor, MI 48109-1043 \\ Email: cvspenc@umich.edu
}

\begin{abstract}
This paper improves on the best-known runtime and measurement bounds for a recently proposed Deterministic sublinear-time Sparse Fourier Transform algorithm (hereafter called DSFT). In [1], [2], it is shown that DSFT can exactly reconstruct the Fourier transform (FT) of an $N$-bandwidth signal $f$, consisting of $B \ll N$ non-zero frequencies, using $O\left(B^{2} \cdot \operatorname{polylog}(N)\right)$ time and $O\left(B^{2} \cdot \operatorname{polylog}(N)\right) f$-samples. DSFT works by taking advantage of natural aliasing phenomena to hash a frequencysparse signal's FT information modulo $O(B \cdot \operatorname{polylog}(N))$ pairwise coprime numbers via $O(B \cdot \operatorname{polylog}(N))$ small Discrete Fourier Transforms. Number theoretic arguments then guarantee the original DFT frequencies/coefficients can be recovered via the Chinese Remainder Theorem. DSFT's usage of primes makes its runtime and signal sample requirements highly dependent on the sizes of sums and products of small primes. Our new bounds utilize analytic number theoretic techniques to generate improved (asymptotic) bounds for DSFT. As a result, we provide better bounds for the sampling complexity/number of low-rate analog-to-digital converters (ADCs) required to deterministically recover frequency-sparse wideband signals via DSFT in signal processing applications [3], [4].
\end{abstract}

Index Terms-Fourier transforms, Discrete Fourier transforms, Algorithms, Number theory, Signal processing

\section{INTRODUCTION}

Compressed Sensing (CS) is an exciting new signal acquisition and recovery paradigm in which highly compressible signals can be (approximately) recovered from a few linear measurements, considerably fewer measurements than previously assumed [5], [6]. This paper will focus on a particular type of compressible signal, namely signals consisting of a small number of significant Fourier modes. Thus, we sample a frequency-sparse signal $f$ on a small deterministic sample set and then reconstruct the signal by returning a list of the predominant frequencies in the spectrum of $f$. This sensing paradigm is useful in many areas, including MR imaging [7], [8], numerical methods for multiscale problems [9], [10], and ADC design [3], [4].

Existing CS (related) Fourier reconstruction algorithms [5], [6], [11], [12], [13] are all either (i) super-linear time in the signal's bandwidth, making them computationally intensive for wideband signals, or (ii) capable of producing incorrect results with some small probability, making them inappropriate for failure intolerant applications. DSFT [1], [2] is both sublineartime and deterministic. Hence, it is an improvement over previous CS (related) Fourier reconstruction algorithms for $N$-bandwidth signals containing $B \ll N$ significant (e.g., nonzero) frequencies, although it does require a $O(B \cdot \operatorname{polylog}(N))$ factor increase in the number of signal samples over previous randomized approaches [14]. Furthermore, DSFT is consistent with recently proposed ADC designs [3], [4] that suggest a radical new approach to analog-to-digital conversion. These ADC designs, which are based on random sampling, currently require the implementation of random clocks, pseudo-random switches, etc. Due to its deterministic nature, DSFT would allow one to build similar circuits with fixed sample sets in the hardware, thus simplifying the circuit design.

In this paper, we employ analytic number theory to give the first asymptotic runtime/sample complexity bounds for DSFT on $B$-support wideband signals (i.e., wideband signals consisting of exactly $B$ non-zero frequencies). Furthermore, we present experiments which both validate our theoretical sample bounds and investigate the number of significant frequencies DSFT may recover from signals of various sizes while maintaining sub-linear sample usage. Finally, we briefly discuss algorithmic improvements which significantly decrease DSFT's sampling and runtime requirements in practice. Our new bounds, besides advancing our knowledge of DSFT's computational properties, also allow us to better bound the number of low-rate parallel ADCs required to deterministically recover wideband frequency-sparse signals along the lines of [3], [4].

\section{Preliminaries}

Throughout the remainder of this paper, we will be interested in complex-valued signals, $f:[0,2 \pi] \rightarrow \mathbb{C}$, which are band-limited and frequency-sparse. Hence, we will assume there exists an $N \in \mathbb{N}$ such that for all our signals $f$,

$$
\left.\Omega_{f}=\{\omega \in \mathbb{Z} \mid \widehat{f}(\omega) \nRightarrow 0\} \subsetneq\left(-\left\lceil\frac{N}{2}\right\rceil, \mid \frac{N}{2}\right\rceil\right] .
$$

Also, we assume that for all $f$, we have $B=\left|\Omega_{f}\right| \ll N$. For any signal $f$, we will refer to the $B$ non-zero elements of $\Omega_{f}$ as $\omega_{1}, \omega_{2}, \ldots, \omega_{B}$. Furthermore, we will refer to the process of either calculating or measuring $f$ at any $t \in[0,2 \pi]$ as sampling from $f$. Finally, we will say that $N$ is $f$ 's bandwidth.

Recently, a Deterministic sublinear-time Sparse Fourier Transform algorithm (DSFT) [1], [2] was developed by 
building upon the number theoretic hashing techniques first proposed in [15], [16]. For a given input signal $f$, DSFT produces output of the form $\left(\omega_{1}, \widehat{f}\left(\omega_{1}\right)\right), \ldots,\left(\omega_{B}, \widehat{f}\left(\omega_{B}\right)\right)$ using $O\left(B^{2} \log ^{7} N\right)$ time and $O\left(B^{2} \log ^{6} N\right)$ samples [1], [2]. In order to get a feel for how DSFT works, consider the following example: Suppose that $f$ is a non-identically zero function of the form $f(x)=C \cdot e^{i \omega x}$ consisting of a single unknown frequency $\omega \in\left(-\frac{N}{2}, \frac{N}{2}\right]$ (e.g., consider a windowed sinusoidal portion of a wideband frequency-hopping signal [3]). Sampling at the Nyquist rate would dictate the need for at least $N$ equally spaced samples from $f$ in order to discover $\omega$ via the Fast Fourier Transform (FFT) without aliasing. Thus, we would have to compute the FFT of the $N$-element vector

$$
\mathbf{A}_{N}(j)=f\left(\frac{2 \pi j}{N}\right), \quad 0 \leq j<N .
$$

However, if we use aliasing to our advantage, we can correctly determine $\omega$ with significantly fewer $f$-samples as follows.

Let $\mathbf{A}_{2}$ be a 2-element array of $f$-samples with

$$
\mathbf{A}_{2}(0)=f(0)=C \text {, and } \mathbf{A}_{2}(1)=f(\pi)=C \cdot(-1)^{\omega} .
$$

Calculating $\widehat{\mathbf{A}_{2}}$, we get that

$$
\widehat{\mathbf{A}_{2}}(0)=C \cdot \frac{1+(-1)^{\omega}}{2} \text { and } \widehat{\mathbf{A}_{2}}(1)=C \cdot \frac{1+(-1)^{\omega+1}}{2} .
$$

Note that since $\omega$ is an integer, exactly one element of $\widehat{\mathbf{A}_{2}}$ will be non-zero. If $\widehat{\mathbf{A}_{2}}(0) \neq 0$, then we know that $\omega \equiv 0$ modulo 2. On the other hand, $\widehat{\mathbf{A}_{2}}(1) \neq 0$ implies that $\omega \equiv 1$ modulo 2 . More generally, if we let $\mathbf{A}_{p}$ be the array

$$
f(0), f\left(\frac{2 \pi}{p}\right), \ldots, f\left(\frac{2 \pi(p-1)}{p}\right),
$$

then

$$
\widehat{\mathbf{A}_{p}}(h)=\frac{C}{p} \cdot \sum_{k=0}^{p-1} e^{\frac{-2 \pi \mathrm{i}(h-\omega) k}{p}}=\left\{\begin{array}{ll}
C & \text { if } h \equiv \omega \bmod p \\
0 & \text { else }
\end{array} .\right.
$$

In this same fashion, we may use several potentially aliased Fast Fourier Transforms in parallel to discover $\omega$ modulo 3, $5, \ldots$, the $O(\log N)^{\text {th }}$ prime. Once we have collected these moduli, we can reconstruct $\omega$ via modular arithmetic. The Chinese Remainder Theorem will guarantee that we identify the correct $\omega$.

Of course, this example is extremely simple. When our input signal $f$ consists of $B>1$ non-zero frequencies, there is the potential for frequencies to collide modulo various primes. Dealing with these potential collisions in a deterministic fashion requires us to hash $f$ 's $B$ frequencies modulo $O(B \cdot \operatorname{polyl} \log (N))$ relatively prime numbers. We next define the numbers which DSFT uses to hash signal frequencies via aliasing. See [1], [2] for details.

Let $p_{0}=1$ and $p_{l}$ be the $l^{\text {th }}$ prime. Next, choose $m$ such that

$$
\prod_{l=1}^{m} p_{l} \geq \frac{N}{B}>\prod_{l=1}^{m-1} p_{l}
$$

Finally, let $q_{K}>\cdots>q_{1}>\max \left(B, p_{m}\right)$ be the smallest $K=3 B\left\lfloor\log _{B} N\right\rfloor+1$ primes $>\max \left(B, p_{m}\right)$. DSFT requires the computation of the Discrete Fourier Transform (DFT) of

$$
f(0), f\left(\frac{2 \pi}{p_{l} q_{j}}\right), \ldots, f\left(\frac{2 \pi\left(p_{l} q_{j}-1\right)}{p_{l} q_{j}}\right)
$$

for every $0 \leq l \leq m$ and $1 \leq j \leq K$. Note that each of these $(m+1) \cdot K$ DFT's will take $O\left(p_{m} q_{K} \log q_{K}\right)$ time despite the factorizations of the array lengths [17], [18].

Fact 1. The number of ADC $f$-samples required by DSFT is

$$
\sum_{l=0}^{m} \sum_{j=1}^{K} p_{l} q_{j}=\left(\sum_{l=0}^{m} p_{l}\right) \cdot\left(\sum_{j=1}^{K} q_{j}\right) .
$$

Fact 2. The runtime of DSFT is

$$
\Theta\left(\sum_{l=0}^{m} \sum_{j=1}^{K} p_{l} q_{j} \log q_{j}\right) .
$$

The remainder of this paper is dedicated to studying (1) and (2). In the following section, we establish necessary lemmas so that in Section IV, we may analyze DSFT's runtime and number of required samples. Then, in Section V, we both empirically validate our Section IV sample bounds for (1) and investigate DSFT's ability to reconstruct superpositions of various sizes using a sub-linear number of samples. Finally, we discuss methods of improving DSFT's sampling performance in Section VI before concluding with a brief discussion in Section VII.

\section{Required Lemmas}

In this section, we will assume that $R \geq 3, B \geq 2$, and $N / B \geq$ 3. Furthermore, $p$ will always stand for a prime number, and $\pi(x)$ will denote the number of primes $\leq x$. The following lemma recalls three forms of the Prime Number Theorem.

Lemma 3. One has that

$$
\pi(x)=\frac{x}{\ln x}+O\left(\frac{x}{\ln ^{2} x}\right)
$$

and that

$$
p_{l}=l \ln l+O(l \ln \ln l) .
$$

Also, there exists a positive constant $c$ such that

$$
\sum_{p \leq x} \ln p=x+O\left(\frac{x}{\exp (c \sqrt{\ln x})}\right) .
$$

We now use the Prime Number Theorem to establish asymptotics required in the analysis of DSFT.

Lemma 4. Choose $m$ such that

$$
\prod_{l=1}^{m} p_{l} \geq \frac{N}{B}>\prod_{l=1}^{m-1} p_{l} .
$$


There exists a positive constant $c$ such that

$$
p_{m}=\ln \frac{N}{B}+O\left(\frac{\ln \frac{N}{B}}{\exp \left(c \sqrt{\ln \ln \frac{N}{B}}\right)}\right) .
$$

Proof: Note that

$$
\prod_{p \leq R} p \geq \frac{N}{B} \quad \text { if and only if } \quad \sum_{p \leq R} \ln p \geq \ln \frac{N}{B} .
$$

It now follows from formula (5) that there exists a positive constant $c$ such that

$$
p_{m}=\ln \frac{N}{B}+O\left(\frac{\ln \frac{N}{B}}{\exp \left(c \sqrt{\ln \ln \frac{N}{B}}\right)}\right) .
$$

Lemma 5. One has

$$
\sum_{p \leq R} p \ln p=\frac{R^{2}}{2}+O\left(\frac{R^{2}}{\ln R}\right) .
$$

Proof: By Riemann-Stieltjes integration (see Chapter 10 of [19]), integration by parts, and formula (3), we obtain

$$
\begin{aligned}
\sum_{p \leq R} p \ln p= & \int_{2^{-}}^{R^{+}}(x \ln x) d \pi(x) \\
= & (R \ln R) \pi(R)-\int_{2}^{R} \pi(x) d(x \ln x) \\
= & R^{2}+O\left(\frac{R^{2}}{\ln R}\right) \\
& -\int_{2}^{R}\left(\frac{x}{\ln x}+O\left(\frac{x}{\ln ^{2} x}\right)\right)(\ln x+1) d x \\
= & \frac{R^{2}}{2}+O\left(\frac{R^{2}}{\ln R}\right) .
\end{aligned}
$$

Lemma 6. One has

$$
\sum_{p \leq R} p=\frac{R^{2}}{2 \ln R}+O\left(\frac{R^{2}}{\ln ^{2} R}\right) .
$$

Proof: Note that

$$
\int_{2}^{\frac{R}{\ln R}} \frac{x}{\ln x} d x=O\left(\frac{R^{2}}{\ln ^{3} R}\right)
$$

and that

$$
\begin{aligned}
\int_{\frac{R}{\ln R}}^{R} \frac{x}{\ln x} d x & =\frac{R^{2}}{\ln R} \int_{\frac{1}{\ln R}}^{1} \frac{y}{1+\frac{\ln y}{\ln R}} d y \\
& =\frac{R^{2}}{\ln R} \int_{\frac{1}{\ln R}}^{1}\left(y-\frac{y \ln y}{\ln R+\ln y}\right) d y \\
& =\frac{R^{2}}{\ln R} \int_{\frac{1}{\ln R}}^{1}\left(y+O\left(\left|\frac{y \ln y}{\ln R}\right|\right)\right) d y \\
& =\frac{R^{2}}{2 \ln R}+O\left(\frac{R^{2}}{\ln ^{2} R}\right) .
\end{aligned}
$$

Therefore, we may conclude that

$$
\int_{2}^{R} \frac{x}{\ln x} d x=\frac{R^{2}}{2 \ln R}+O\left(\frac{R^{2}}{\ln ^{2} R}\right) .
$$

By Riemann-Stieltjes integration, integration by parts, and formulas (3) and (6), it follows that

$$
\begin{aligned}
\sum_{p \leq R} p= & \int_{2^{-}}^{R^{+}} x d \pi(x)=R \pi(R)-\int_{2}^{R} \pi(x) d x \\
= & \frac{R^{2}}{\ln R}+O\left(\frac{R^{2}}{\ln ^{2} R}\right) \\
& -\int_{2}^{R}\left(\frac{x}{\ln x}+O\left(\frac{x}{\ln ^{2} x}\right)\right) d x \\
= & \frac{R^{2}}{2 \ln R}+O\left(\frac{R^{2}}{\ln ^{2} R}\right) .
\end{aligned}
$$

IV. Runtime and Measurement Bounds

We now are prepared to analyze the performance of DSFT. By formula (3), we have

$$
\pi\left(\max \left(B, p_{m}\right)\right)=O\left(\frac{B}{\ln B}+\frac{\ln \frac{N}{B}}{\ln \ln \frac{N}{B}}\right) .
$$

When

$$
\begin{aligned}
S & =\pi\left(\max \left(B, p_{m}\right)\right)+K \\
& =3 B\left\lfloor\log _{B} N\right\rfloor+O\left(\frac{B}{\ln B}+\frac{\ln \frac{N}{B}}{\ln \ln \frac{N}{B}}\right),
\end{aligned}
$$

then by formula (4), we have

$$
\begin{aligned}
q_{K} & =p_{S}=S \ln S+O(S \ln \ln S) \\
& =3 B\left\lfloor\log _{B} N\right\rfloor \ln (B \ln N)\left(1+O\left(\frac{\ln \ln (B \ln N)}{\ln (B \ln N)}\right)\right) .
\end{aligned}
$$

Using Lemma 6, we see that

$$
\begin{aligned}
\sum_{j=1}^{K} q_{j} & =\sum_{\max \left(B, p_{m}\right)<p \leq q_{K}} p \\
& =\frac{9}{2} B^{2}\left\lfloor\log _{B} N\right\rfloor^{2} \ln (B \ln N)\left(1+O\left(\frac{\ln \ln (B \ln N)}{\ln (B \ln N)}\right)\right)
\end{aligned}
$$

and

$$
\begin{aligned}
\sum_{l=0}^{m} p_{l} & =\frac{p_{m}^{2}}{2 \ln p_{m}}+O\left(\frac{p_{m}^{2}}{\ln ^{2} p_{m}}\right) \\
& =\frac{\ln ^{2} \frac{N}{B}}{2 \ln \ln \frac{N}{B}}\left(1+O\left(\frac{1}{\ln \ln \frac{N}{B}}\right)\right) .
\end{aligned}
$$

Combining the above two estimates with Fact 1, we obtain the following theorem.

Theorem 7. The number of $f$-samples required by DSFT is

$$
\begin{aligned}
\frac{9 B^{2}\left\lfloor\log _{B} N\right\rfloor^{2} \ln (B \ln N) \ln ^{2} \frac{N}{B}}{4 \ln \ln \frac{N}{B}} \times \\
\left(1+O\left(\frac{1}{\ln \ln \frac{N}{B}}+\frac{\ln \ln (B \ln N)}{\ln (B \ln N)}\right)\right) .
\end{aligned}
$$


By Lemma 5, we have

$$
\begin{aligned}
\sum_{j=1}^{K} q_{j} \ln q_{j} & =\sum_{\max \left(B, p_{m}\right)<p \leq q_{K}} p \ln p \\
& =\frac{q_{K}^{2}}{2}+O\left(\frac{q_{K}^{2}}{\ln q_{K}}+B^{2}+p_{m}^{2}\right) \\
& =\frac{\left(3 B\left\lfloor\log _{B} N\right\rfloor \ln (B \ln N)\right)^{2}}{2}\left(1+O\left(\frac{\ln \ln (B \ln N)}{\ln (B \ln N)}\right)\right) .
\end{aligned}
$$

By combining Fact 2 with formulas (7) and (8), we obtain the following theorem.

Theorem 8. The runtime of DSFT is

$$
\Theta\left(B^{2} \cdot \frac{\ln ^{2} N \cdot \ln ^{2} \frac{N}{B} \cdot \ln ^{2}(B \ln N)}{\ln ^{2} B \cdot \ln \ln \frac{N}{B}}\right) .
$$

Let $\alpha \in\left(0, \frac{1}{2}\right)$ be a constant, and suppose that $B=$ $\Theta\left(N^{\alpha}\right)$. In this case, we have improved the previous best sample bound for DSFT from $O\left(B^{2} \log ^{6} N\right)$ to $\Theta\left(B^{2} \cdot \frac{\log ^{3} N}{\log \log N}\right)$. Furthermore, we have improved the previous best bound for DSFT's runtime from $O\left(B^{2} \log ^{7} N\right)$ to $\Theta\left(B^{2} \cdot \frac{\log ^{4} N}{\log \log N}\right)$. Finally, in signal processing applications along the lines of [3], [4], we can see that the sub-Nyquist sampling required to compute DSFT's $(m+1) \cdot K$ DFTs can be accomplished via $(m+1) \cdot K=O\left(B \cdot \frac{\log N}{\log \log N}\right)$ parallel analog-to-digital converters, each with rate $O\left(p_{m} q_{K}\right)=O\left(B \log ^{2} N\right) \mathrm{Hz}$. Hence, DSFT provides a promising deterministic method for quickly reconstructing frequency-sparse wideband signals. We next empirically investigate the signal sizes for which DSFT (as formulated in [1]) can reconstruct sparse superpositions with sub-linear sampling requirements.

\section{Sampling: Empirical Evaluation}

In order to test DSFT's sample asymptotic (Theorem 7), we compare the number of $f$-samples DSFT requires to perfectly recover an $N$-bandwidth signal $f$ containing exactly $B=512$ non-zero frequencies against the sample asymptotic's main term in Theorem 7. The number of DSFT samples required to recover a 512 -frequency superposition, divided by the associated asymptotic value in Theorem 7, is plotted in Figure 1 for various bandwidth values $N$. Figure 1 demonstrates that our asymptotic is within a constant multiple of 2 of the true number of samples required by DSFT for all bandwidth values. Thus, despite the fact that our asymptotic converges to DSFT's number of utilized samples at an exceedingly slow pace, it appears as if the asymptotic generally gives us a reliable estimate of DSFT's sampling requirements. Experiments performed for smaller $B$ reinforce this observation.

Given that a standard FFT can determine the Fourier transform of an $N$-bandwidth signal $f$ by taking $N$ samples from $f$, it is important for us to determine when DSFT enables us to utilize less than $N$ samples to recover $\widehat{f}$. Figure 2 addresses this issue by plotting, for each bandwidth value $N$,

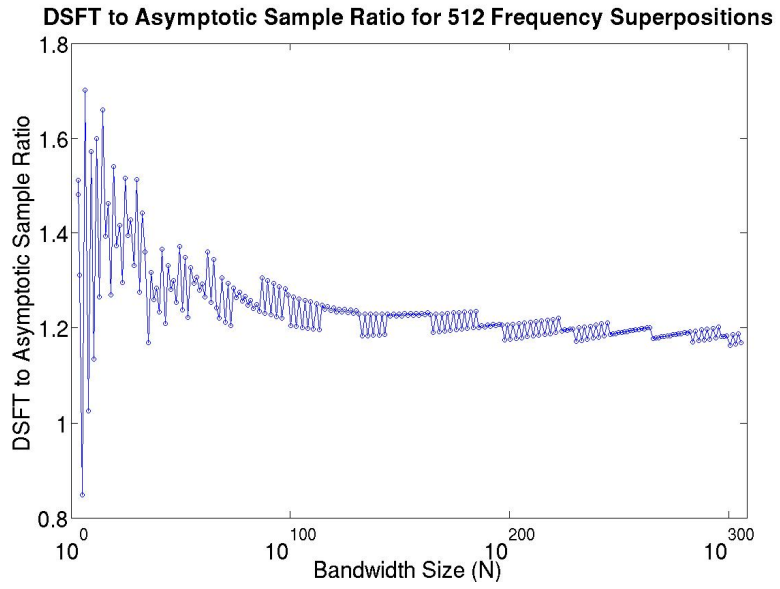

Fig. 1. Empirical Test of Theorem 7.

the maximum number of non-zero frequencies $f$ may contain while still allowing DSFT to determine $\widehat{f}$ using less than $N$ $f$-samples. Figure 2 demonstrates that DSFT, as formulated

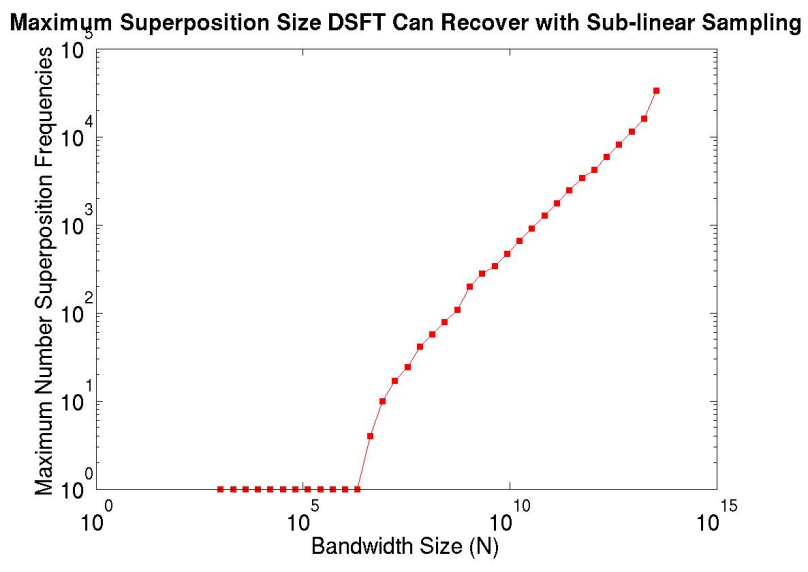

Fig. 2. Maximum $B$ Value Yielding Less Than $N$ Samples.

in [1] and [2], does not exhibit sub-linear sampling for $B>1$ until the bandwidth is $\geq 2^{22}$ (about 4 million). In Section VI, we will discuss improvements/modifications for DSFT which allow sub-linear sampling for significantly smaller signals.

Finally, Figure 3 plots the number of $f$-samples DSFT requires to recover $\widehat{f}$ divided by $f$ 's bandwidth, $N$, for three different bandwidth values. It is interesting to note that DSFT's number of required samples occasionally decreases as $B$ increases. This is due to $K=3 B\left\lfloor\log _{B} N\right\rfloor+1$ decreasing in size, implying that DSFT requires fewer $q_{j}$-primes (see Section II). We will use this phenomenon to our advantage in the following section.

\section{SAMPLing: Improving DSFT's Performance}

DSFT's sample usage can be mildly decreased (i.e., by a constant factor) through a more careful choice of which primes $p_{l}$ and $q_{j}$ from Section II are used for sampling. In 


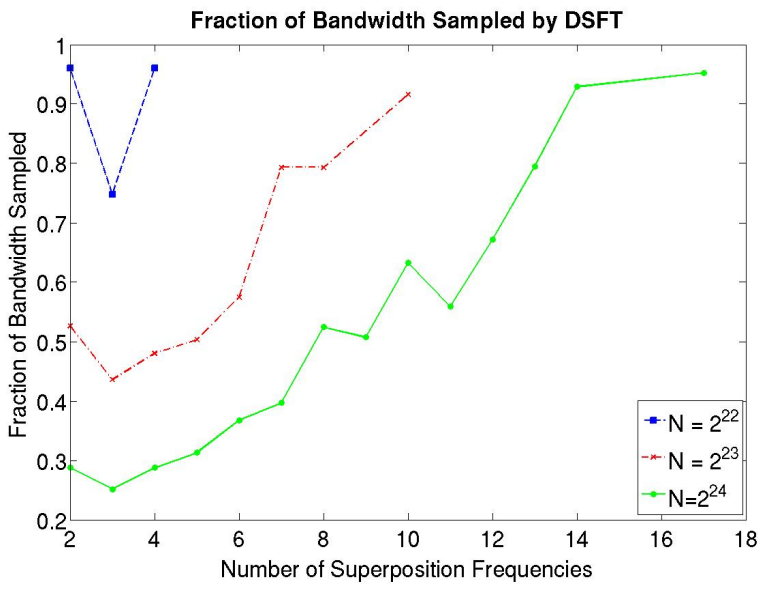

Fig. 3. Fraction of Bandwidth Sampled for Various $B$ Values.

this section, we will discuss three such DSFT improvements. We will also briefly mention two more radical changes to DSFT which dramatically reduce the sample usage, but at the expense of either loosing DSFT's sub-linear reconstruction time or deterministic nature.

First, it should be noted that using powers of $p_{l}$-primes can decrease DSFT's sample usage. Instead of performing DFTs of size $p_{0} \cdot q_{j}, \ldots, p_{m} \cdot q_{j}$ for each $q_{j}$-prime, one can implement DSFT using DFTs of size

$$
p_{0} \cdot q_{j}, p_{1}^{\alpha_{j, 1}} \cdot q_{j}, \ldots, p_{v}^{\alpha_{j, v}} \cdot q_{j}, \ldots, p_{m_{j}}^{\alpha_{j, m_{j}}} \cdot q_{j}
$$

for each $q_{j}$-prime (see Section II). This would require that

$$
\prod_{v=1}^{m_{j}} p_{v}^{\alpha_{j, v}} \geq \frac{N}{q_{j}}>\prod_{v=1}^{m_{j}-1} p_{v}^{\alpha_{j, v}}
$$

for each $q_{j}$. We would then replace each

$$
\left(\sum_{l=0}^{m} p_{l}\right) \cdot q_{j} \quad \text { term with a }\left(\sum_{v=0}^{m_{j}} p_{v}^{\alpha_{j, v}}\right) \cdot q_{j} \quad \text { term }
$$

in our bounds for the number of $f$-samples and the runtime. Finally, the condition that $q_{1}>\max \left(B, p_{m}\right)$ would be replaced with the requirement that $q_{j}>\max \left(B, p_{m_{j}}\right)$ for each $1 \leq j \leq K$.

Second, as pointed out at the end of the last section, using a larger $B$ for DSFT sometimes decreases sampling requirements (see Figure 3). We may use this phenomenon to our advantage by increasing the size of $q_{1}$ and redefining our required number of $q_{j}$-primes to be

$$
K=3 B\left\lfloor\log _{q_{1}} N\right\rfloor+1 .
$$

Here $q_{1}>\max \left(B, p_{m_{1}}\right)$, as in the previous paragraph, but $q_{1}$ need not be the smallest prime $>\max \left(B, p_{m_{1}}\right)$. By altering $q_{1}$ and $K$ 's definitions in this fashion, it becomes clear that slightly increasing $q_{1}$ can be beneficial.

Third, by a careful analysis of the arguments in [1], [2], DSFT can be modified to require only $K=2 B\left\lfloor\log _{q_{1}} N\right\rfloor+1$ $q_{j}$-primes (i.e., $K$ 's factor of 3 can be reduced to a 2 ) while still maintaining its sub-linear $\tilde{\Theta}\left(B^{2}\right)$-runtime in Theorem 8 .
No modification of the $p_{v}{ }^{\alpha_{j, v}}$-values are required. We will next consider an example that demonstrates the utility of these three modifications with respect to DSFT's sample usage.

Consider an $N=50,000$ bandwidth signal $f$ containing exactly $B=5$ non-zero frequencies. DSFT, as formulated in Section II, [1], and [2], would require almost 950, 000 samples to recover $\widehat{f}$. DSFT would use $2,3,5,7,11$, and 13 as its $p_{l^{-}}$ primes, leading to a total of

$$
41 \cdot\left(\sum_{j=1}^{K} q_{j}\right)
$$

DSFT samples. However, if we require $q_{1}$ to be greater than

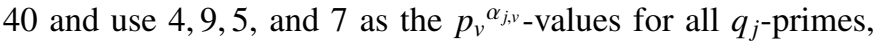
we can modestly reduce DSFT's sample usage to

$$
25 \cdot\left(\sum_{j=1}^{K} q_{j}\right) .
$$

In addition to our savings from using different $p_{v}{ }^{\alpha_{j, v}}$-values, using $q_{1}=41$ instead of $q_{1}=11$ also allows the use of fewer $q_{j}$-primes (see (9)). This, in combination with the aforementioned algorithmic reduction of $K$ 's constant factor from 3 to 2 , will allow a well optimized DSFT implementation to recover our $N=50,000$ bandwidth, 5-frequency superposition $f$ using roughly 43,000 samples (about 22 times fewer samples than previously required). Thus, optimizing DSFT can dramatically improve its performance.

Further reductions in DSFT's sampling requirements can be obtained if the user is willing to tolerate a super-linear $\tilde{O}(B \cdot N)$ Fourier reconstruction time for $N$-bandwidth signals with $B$ non-zero frequencies. After performing DFT's of length $q_{1}, q_{2}, \ldots, q_{K}$, as in Section II, one can determine the Fourier coefficient for any of the signal's $N$, possibly non-zero, frequencies as follows: For each

$$
\omega \in\left(-\left\lceil\frac{N}{2}\right\rceil,\left\lfloor\frac{N}{2}\right\rfloor\right],
$$

we first determine $\omega$ 's residue modulo $q_{j}$ for each $q_{j}$. Proofs analogous to those in [1], [2] then guarantee that $\omega$ 's Fourier coefficient's real/imaginary part will equal the median of the real/imaginary parts of the $K$ DFT entries associated with $\omega$ 's residues modulo each $q_{j}$. Thus, we no longer need any $p_{v}{ }^{\alpha_{j, v}}$ values if we are willing to inspect all $N$ frequencies in this fashion. The number of required samples is reduced to

$$
\sum_{j=1}^{K} q_{j} .
$$

Returning to the last paragraph's example, this modified DSFT method only needs 1,791 samples to correctly recover an $N=50,000$ bandwidth, $B=5$ superposition's Fourier transform. This represents roughly an additional 24-fold decrease in DSFT's sampling needs. However, we are forced to abandon DSFT's sub-linear runtime.

Finally, it is also worthwhile to note that Monte Carlo Fourier results similar to those of [14] may be obtained by limiting our $q_{j}$-prime usage in Section II. If we only use a 
small subset of randomly chosen $q_{j}$, we will still be able to isolate all non-zero superposition frequencies with high probability. The frequency's coefficients can then either $(i)$ be approximated by USFFT techniques [14], [20], [21], [22] or (ii) be recovered exactly (assuming non-zero frequency isolation occurs more often then not) using a procedure similar to the one outlined in the previous paragraph. This allows one to use only $\tilde{O}(B)$-samples/runtime for $B$-frequency superposition reconstruction, which is within a polylogarithmic factor of the current best sample bounds for sparse signal reconstruction via Linear Programming [23], [24]. However, modifying our DSFT techniques in this fashion only allows one to reconstruct sparse superpositions with high probability, and the deterministic nature of our algorithm is lost.

\section{Conclusion}

In this paper, we utilized analytic number theory to develop the first known asymptotic runtime/sample complexity bounds for DSFT on $B$-support wideband signals. We then empirically evaluated our new DSFT sampling bounds in Section V. Let $\alpha \in\left(0, \frac{1}{2}\right)$ be a constant, and suppose that $B=\Theta\left(N^{\alpha}\right)$. In this case, we have improved the previous best sample bound for DSFT from $O\left(B^{2} \log ^{6} N\right)$ to $\Theta\left(B^{2} \cdot \frac{\log ^{3} N}{\log \log N}\right)$. Furthermore, we have improved the previous best bound for DSFT's runtime from $O\left(B^{2} \log ^{7} N\right)$ to $\Theta\left(B^{2} \cdot \frac{\log ^{4} N}{\log \log N}\right)$. In Section VI, we demonstrated that if one is willing to tolerate a super-linear $O\left(B\left\lfloor\log _{q_{1}} N\right\rfloor \log \left(B\left\lfloor\log _{q_{1}} N\right\rfloor\right) \cdot N\right)$ reconstruction runtime (after all DFTs have been taken), then DSFT's sampling bound can be reduced to $\Theta\left(B^{2} \cdot \log N\right)$. Furthermore, if one is willing to exchange determinism for success with high probability, we can reduce both DSFT's runtime and sampling needs to $O(B \cdot \operatorname{polylog}(N))$.

In signal processing applications [3], [4], we have shown that the sub-Nyquist sampling required to compute DSFT's $(m+1) \cdot K$ DFTs can be accomplished via $(m+1) \cdot K=$ $O\left(B \cdot \frac{\log N}{\log \log N}\right)$ parallel ADCs, each with rate $O\left(B \log ^{2} N\right)$ Hz. Hence, DSFT provides a promising deterministic method for quickly reconstructing frequency-sparse wideband signals. Finally, it is worth noting that our DSFT methods are closely related to combinatorial group testing and many other algorithmic problems involving hashing by consecutive primes [15], [25]. Our new DSFT bounds should also provide asymptotic bounds for these related methods.

\section{ACKNOWLEDGMENTS}

The authors would like to thank Anna Gilbert, Martin Strauss, Mike Lieberman, and Wendy Grus for helpful comments and advice. This work was supported in part by NSF grants DMS-0510203 and DMS-0601367.

\section{REFERENCES}

[1] M. A. Iwen, "A deterministic sub-linear time sparse fourier algorithm via non-adaptive compressed sensing methods," in Proc. of ACM-SIAM symposium on Discrete algorithms (SODA'08), 2008.

[2] M. A. Iwen, "A deterministic sub-linear time sparse fourier algorithm via non-adaptive compressed sensing methods," ArXiv Computer Science e-prints, Aug. 2007.

[3] Jason Laska, Sami Kirolos, Yehia Massoud, Richard Baraniuk, Anna Gilbert, Mark Iwen, and Martin Strauss, "Random sampling for analogto-information conversion of wideband signals," Proc. IEEE Dallas Circuits and Systems Conference, 2006.

[4] Sami Kirolos, Jason Laska, Michael Wakin, Marco Duarte, Dror Baron, Tamer Ragheb, Yehia Massoud, and Richard Baraniuk, "Analog-toinformation conversion via random demodulation," Proc. IEEE Dallas Circuits and Systems Conference, 2006.

[5] D. Donoho, "Compressed Sensing," IEEE Trans. on Information Theory, vol. 52, pp. 1289-1306, 2006.

[6] E. Candes, J. Romberg, and T. Tao, "Robust uncertainty principles: Exact signal reconstruction from highly incomplete frequency information," IEEE Trans. Inform. Theory, vol. 52, pp. 489-509, 2006.

[7] M. Lustig, D. Donoho, and J. Pauly, "Sparse MRI: The application of compressed sensing for rapid MR imaging," Submitted for publication, 2007.

[8] R. Maleh, A. C. Gilbert, and M. J. Strauss, "Signal recovery from partial information via orthogonal matching pursuit," IEEE Int. Conf. on Image Processing, 2007.

[9] I. Daubechies, O. Runborg, and J. Zou, "A sparse spectral method for homogenization multiscale problems," Multiscale Model. Sim., 2007.

[10] M. A. Iwen, "Unpublished Results," http://wwwpersonal.umich.edu/ markiwen/.

[11] R. A. DeVore, "Deterministic constructions of compressed sensing matrices," http://www.ima.umn.edu/2006-2007/ND6.415.07/activities/DeVore-Ronald/Henrykfinal.pdf, 2007.

[12] J. Tropp and A. Gilbert, "Signal recovery from partial information via orthogonal matching pursuit," Submitted for Publication, 2005.

[13] A. Gilbert, S. Guha, P. Indyk, S. Muthukrishnan, and M. Strauss, "Nearoptimal sparse Fourier estimation via sampling," ACM STOC, pp. 152$161,2002$.

[14] A. Gilbert, S. Muthukrishnan, and M. Strauss, "Improved time bounds for near-optimal sparse Fourier representations," SPIE, 2005.

[15] S. Muthukrishnan, "Data Streams: Algorithms and Applications," Foundations and Trends in Theoretical Computer Science, vol. 1, 2005.

[16] G. Cormode and S. Muthukrishnan, "Combinatorial Algorithms for Compressed Sensing," Technical Report DIMACS TR 2005-40, 2005.

[17] Leo I. Bluestein, "A Linear Filtering Approach to the Computation of Discrete Fourier Transform," IEEE Transactions on Audio and Electroacoustics, vol. 18, pp. 451-455, 1970.

[18] L.R. Rabiner, R.W. Schafer, and C.M. Rader, "The Chirp z-Transform Algorithm," IEEE Transactions on Audio and Electroacoustics, vol. AU-17, no. 2, pp. 86-92, June 1969.

[19] N. B. Haaser and J. A Sullivan, Real analysis, Dover Publications, Inc., 1991.

[20] A. Dutt and V. Rokhlin, "Fast Fourier transforms for nonequispaced data," SIAM J. Sci. Comput., vol. 14, pp. 1368-1383, 1993.

[21] J. A. Fessler and B. P. Sutton, "Nonuniform Fast fourier transforms using min-max interpolation," IEEE Trans. Signal Proc., vol. 51, pp. 560-574, 2003.

[22] J.-Y. Lee and L. Greengard, "The type 3 nonuniform FFT and its applications," J Comput. Phys., vol. 206, pp. 1-5, 2005.

[23] Mark Rudelson and Roman Vershynin, "Sparse reconstruction by convex relaxation: Fourier and gaussian measurements," in 40th Annual Conference on Information Sciences and Systems (CISS), 2006.

[24] David L. Donoho and Jared Tanner, "Thresholds for the recovery of sparse solutions via 11 minimization," in 40th Annual Conference on Information Sciences and Systems (CISS), 2006.

[25] S. Ganguly and A. Majumder, "CR-precis: A deterministic summary structure for update data streams," ArXiv Computer Science e-prints, Sept. 2006. 